\title{
Parametric vibrations and stability of viscoelastic shells
}

\author{
M.H. Ilyasov
}

Received: 6 June 2007 / Accepted: 7 October 2009 / Published online: 4 March 2010

(C) The Author(s) 2010. This article is published with open access at Springerlink.com

\begin{abstract}
The problem of dynamic stability of viscoelastic extremely shallow and circular cylindrical shells with any hereditary properties, including time-dependence of Poisson's ratio, are reduced to the investigation of stability of the zero solution of an ordinary integro-differential equation with variable coefficients. Using the Laplace integral transform, an integro-differential equation is reduced to the new integro-differential one of which the main part coincides with the damped Hill equation and the integral part is proportional to the product of two small parameters. Changing this equation for the system of two linear equations of the first order and using the averaging method, the monodromy matrix of the obtained system is constructed. Considering the absolute value of the eigen-values of monodromy matrix is greater than unit, the condition for instability of zero solution is obtained in the three-dimensional space of parameters corresponding to the frequency, viscosity and amplitude of external action. Analysis of form and size of instability domains is carried out.
\end{abstract}

Keywords Viscoelastic shells · Any hereditary property · Time-dependent Poisson ratio · Damped Hill equation · Monodromy matrix $\cdot$ Stability $\cdot$ Resonance

\section{Introduction}

Analysis of the parametric vibrations of the elastic systems subjected to time varying loadings is one of the well-established areas of applied mechanics (Bolotin 1964; Seyranyan and Mailybaev 2003). Adopting elastic theory for the solution of the structures that are made of polymer composites proves to be inconsistent with reality. The viscoelastic theory appears to be suitable for describing the behavior of a large group of materials. Some important results for dynamic stability of viscoelastic materials are obtained (Bolotin 1964; Seyranyan and Mailybaev 2003; Ilyasova 2002-2003; Ilyasov and Aköz 2000; Ahmadi and Glockner 1983; Wang and Lakes 2005; Pata 2006; Shuping et al. 1998; Ilyasov 2007;

M.H. Ilyasov ( $\varangle)$

Azerbaijan National Academy of Sciences, Institute of Mathematics and Mechanics, Khanlar st. 24/44,

Sabail district, Baku 1003, Azerbaijan

e-mail: mhan@istanbul.edu.tr 
Giorgi and Lazzai 1997; Liu and Zheng 1996). Damping plays the main role in the problem of dynamic stability of elastic systems. It is known that if the construction element is loaded by periodic forces in their midsurface, instability occurs for some frequency and amplitude of the applied force. The monodromy matrix method is used in Seyranyan and Mailybaev (2003), Ilyasova (2002-2003) for determination of stability regions of Hill's equation. The influence of viscoelasticity on the instability region is investigated in Ilyasov and Aköz (2000) and it is found that viscoelasticity increases the stability of the plate. The problem of dynamic stability of Kelvin-viscoelastic column is studied in Ahmadi and Glockner (1983). The stability of the discrete viscoelastic system with negative stiffness elements is investigated in Wang and Lakes (2005). The asymptotic behavior of solutions of an abstract integro-differential equation modeling linear viscoelasticity is investigated in Pata (2006). Asymptotic and exponential stabilities for the Kelvin-Voigt damping are investigated in Shuping et al. (1998). A new method for investigation of dynamic stability of viscoelastic plates for any hereditary properties, including time-dependence of Poisson's ratio, is proposed in Ilyasov (2007). The condition for instability of zero solution of obtained integro-differential equation is derived and investigated in the three-dimensional space of parameters.

In order to simplify the mathematical difficulties for the solution of multidimensional dynamical problems for viscoelastic material the viscoelastic Poisson ratio is assumed to be constant. This approach reduces to misstatements of the problems and erroneous of the obtained results. As it has been shown in Tschoegl et al. (2002), the viscoelastic Poisson ratio is not constant except for incompressible materials. In current paper, the dynamic stability of shells, with general isotropic viscoelastic constitutive equations are examined. Using equations known for elastic shells (Bolotin 1964), the equations of parametric vibrations of both extremely shallow and circular cylindrical shells are derived for any viscoelastic functions including time-dependence of Poisson's ratio. By the method of separation of variables, the problems are reduced to the investigation of stability of the zero solution of the ordinary integro-differential equation with variable coefficient. The equation is reduced to a new integro-differential equation for which the main part is the damped Hill equation and the integral part is proportional to the product of two small parameters. The monodromy matrix of the obtained equation and its eigenvalues are constructed. Supposing the absolute value of eigenvalue is greater than the unit, the condition of instability is obtained. Analysis of instability regions with respect to parameters is carried out. Examples have been considered.

\section{Statement of the problem}

In this section we will derive the equations of parametric vibrations of isotropic homogeneous viscoelastic shells for any real hereditary properties. We will consider both the extremely shallow and the circular cylindrical shells with constant thickness.

\subsection{Extremely shallow shell}

Let us assume that in the plan view the shell has the form of a rectangle with sides $a$ and $b$ and, moreover, that the rise of the shell is relatively small in comparison with the sides of the rectangle. Let $x, y$ be the Cartesian coordinates of a point on the horizontal plane. We assume that the principal curvatures of the middle plane in the directions of axes $0 x$ and $0 y$ are $k_{1}=$ const, $k_{2}=$ const. Furthermore, for the case of a shallow shell, the tangential 
components of the load can be neglected. Under these assumptions, the dynamic stability of elastic shell is reduced in Bolotin (1964) to the equation

$$
m \frac{\partial^{2}}{\partial t^{2}} \nabla^{2} \nabla^{2} F+\left(N_{1} \frac{\partial^{2}}{\partial x^{2}}+N_{2} \frac{\partial^{2}}{\partial y^{2}}\right) \nabla^{2} \nabla^{2} F+E h \nabla_{k}^{2} \nabla_{k}^{2} F+D \nabla^{2} \nabla^{2} \nabla^{2} \nabla^{2} F=0,
$$

where

$$
\nabla^{2}=\frac{\partial^{2}}{\partial x^{2}}+\frac{\partial^{2}}{\partial y^{2}}, \quad \nabla_{k}^{2}=k_{2} \frac{\partial^{2}}{\partial x^{2}}+k_{1} \frac{\partial^{2}}{\partial y^{2}},
$$

$m$ is the mass of the shell per unit area of the middle surface, $D=E h^{3} / 12\left(1-v^{2}\right)$ is the flexural stiffness of the shell, $E$ is Young modulus, $v$ is Poisson ratio, $h$ is thickness of the shell, the function $F$ is related to the normal displacement $w$ by the formula $w=\nabla^{2} \nabla^{2} F$, $N_{1}$ and $N_{2}$ are the normal forces on the middle surface of the shell, which we will take as

$$
N_{j}=N_{j 0}-N_{j 1} \varphi(t) \quad(j=1,2)
$$

where $N_{j 0}, N_{j 1}$ are constants and $\varphi(t)$ is $T$-periodic function.

Assume that the shell is simply supported on all four sides. In this case the function $F(t, x, y)$ must be chosen in such a way that

$$
\begin{aligned}
& F=\frac{\partial^{2} F}{\partial x^{2}}=\frac{\partial^{4} F}{\partial x^{4}}=\frac{\partial^{6} F}{\partial x^{6}}=0 \quad \text { at } x=0 \text { and } x=a, \\
& F=\frac{\partial^{2} F}{\partial y^{2}}=\frac{\partial^{4} F}{\partial y^{4}}=\frac{\partial^{6} F}{\partial y^{6}}=0 \quad \text { at } y=0 \text { and } y=b .
\end{aligned}
$$

To write (1) for viscoelastic shell we use the elastic-viscoelastic correspondence principle between the Laplace-Carson transform of elastic and viscoelastic problems (Tschoegl et al. 2002; Ilyasov and Ilyasova 2006; Iliushin and Pobedrya 1970; Christensen 1971). As it is seen from (1), for viscoelastic shell we should invert the functions $\bar{E} \bar{f}$ and $\frac{\bar{E}}{1-\bar{v}^{2}} \bar{f}$, where the bar above the functions denotes its Laplace-Carson transform $\bar{f}(s)=s \int_{0}^{\infty} f(t) e^{-s t} d t$ and $s$ is the complex parameter of the transformation. We will describe these functions by means of shear and dilatation relaxation functions $G(t)$ and $K(t)$, respectively, and corresponding creep functions. It is known that these functions are monotonically decreasing continuous functions, having monotonically increasing integrable derivatives on $t>0$, moreover, $0<$ $G(\infty)=G_{\infty} \leq G(t) \leq G(0)=G$ and $0<K(\infty)=K_{\infty} \leq K(t) \leq K(0)=K$ where $G_{\infty}$, $K_{\infty}$ and $G, K$ are the equilibrium and instantaneous elastic moduli, respectively and $G(t)=$ $K(t) \equiv 0$ for $t<0$ (Tschoegl et al. 2002; Iliushin and Pobedrya 1970; Christensen 1971).

Using the relations

$$
\bar{E}=\frac{9 \bar{G} \bar{K}}{\bar{G}+3 \bar{K}}, \quad \bar{v}=\frac{3 \bar{K}-2 \bar{G}}{2(\bar{G}+3 \bar{K})}
$$

it is easy to get

$$
\frac{\bar{E}}{1-\bar{v}^{2}}=\frac{4 \bar{G}(\bar{G}+3 \bar{K})}{3 \bar{K}+4 \bar{G}}=\bar{G}+3 \bar{G} \bar{g}_{2}=\bar{N},
$$

where $g_{2}(t)$ is the Iliushin creep function with the initial value $g_{2}(0)=(1+v) / 3(1-v)$ (Iliushin and Pobedrya 1970). Here $E$ and $v$ denote the initial value of the functions $E(t)$ and $v(t)$, respectively. The function $g_{2}(t)$ can be obtained both theoretically and directly by 
the experiment (Iliushin and Pobedrya 1970). Let $\bar{\omega}=2 \bar{G} / 3 \bar{K}=(1-2 \bar{v}) /(1+\bar{v})$, then we have $\omega(t)=\frac{2}{3 K} G(t)+\frac{2}{3} \int_{0+}^{t} G(t-\tau) d J_{1}(\tau)$ and if $\bar{K} \equiv K$ is constant, $\omega(t)=\frac{2}{3 K} G(t)$ is obtained. Here $J_{1}(t)\left(J_{1}(0)=1 / K\right)$ is the dilatation creep function.

It is easy to verify the equalities $\omega_{\max }=\bar{\omega}_{\max }=\omega_{0}=(1-2 v) /(1+v) \approx 1 / 4$ and $\omega_{\min }=$ $\bar{\omega}_{\min }=\omega_{0} G(\infty) / G>0$, here $|2 \bar{\omega}|<1$ is obtained So we have the absolutely and uniformly convergent series

$$
\bar{g}_{2}=\frac{1}{1+2 \bar{\omega}}=1-2 \bar{\omega}+4 \bar{\omega}^{2}-\cdots,
$$

and the series expression of the function $g_{2}(t)$

$$
g_{2}(t)=1-2 \omega(t)+4\left[\omega_{0} \omega(t)+\int_{0+}^{t} \omega(t-\tau) d \omega(\tau)\right]-\cdots .
$$

The inversion

$$
N(t)=2 G(t) /(1-v)+3 \int_{0+}^{t} G(t-\tau) d g_{2}(\tau)
$$

and

$$
\frac{\bar{E}}{1-\bar{v}^{2}} \bar{f}=\bar{N} \bar{f} \doteqdot N(0)\left[f(t)+\frac{1}{N(0)} \int_{0}^{t} N^{\prime}(t-\tau) f(\tau) d \tau\right]
$$

are easily obtained. It is seen that $N(0)=E /\left(1-v^{2}\right)$. Let us denote $\Gamma_{3}(t)=-N^{\prime}(t) / N(0)$, then we write

$$
\frac{\bar{E}}{1-\bar{v}^{2}} \bar{f} \doteqdot \frac{E}{1-v^{2}}\left[f(t)-\int_{0}^{t} \Gamma_{3}(t-\tau) f(\tau) d \tau\right] .
$$

By the same way we find

$$
\bar{E}=2 \bar{G}(1+\bar{v})=3 \bar{G} \frac{1}{1+\bar{\omega} / 2} \doteqdot 2(1+v) G(t)+3 \int_{0+}^{t} G(t-\tau) d g_{1 / 2}(\tau)=E(t)
$$

where

$$
g_{1 / 2}(t)=1-\frac{1}{2} \omega(t)+\frac{1}{4}\left[\omega_{0} \omega(t)+\int_{0+}^{t} \omega(t-\tau) d \omega(\tau)\right]-\cdots
$$

Then

$$
\bar{E} \bar{f} \doteqdot E\left[f(t)+\frac{1}{E} \int_{0}^{t} E^{\prime}(t-\tau) f(\tau) d \tau\right]
$$

and denoting $\Gamma_{2}(t)=-E^{\prime}(t) / E$, we write

$$
\bar{E} \bar{f} \doteqdot E\left[f(t)-\int_{0}^{t} \Gamma_{2}(t-\tau) f(\tau) d \tau\right]
$$

If Poisson's ratio is constant then $N(t)=2 G(t) /(1-v)$. The relation between the functions $\Gamma_{2}(t), \Gamma_{3}(t)$ and the shear relaxation function $G(t)$ is obtained as $\Gamma_{2}(t)=\Gamma_{3}(t)=$ $-G^{\prime}(t) / G=\Gamma_{1}(t)$. 
Using (3) and (4), from (1) we obtain the equation of motion for viscoelastic shell for any (satisfying only the natural conditions)hereditary property

$$
\begin{aligned}
& m \frac{\partial^{2}}{\partial t^{2}} \nabla^{2} \nabla^{2} F+\left(N_{1} \frac{\partial^{2}}{\partial x^{2}}+N_{2} \frac{\partial^{2}}{\partial y^{2}}\right) \nabla^{2} \nabla^{2} F+E h \nabla_{k}^{2} \nabla_{k}^{2} F+D \nabla^{2} \nabla^{2} \nabla^{2} \nabla^{2} F \\
& \quad=E h \int_{0}^{t} \Gamma_{2}(t-\tau) \nabla_{k}^{2} \nabla_{k}^{2} F(\tau, x, y) d \tau+D \int_{0}^{t} \Gamma_{3}(t-\tau) \nabla^{2} \nabla^{2} \nabla^{2} \nabla^{2} F(\tau, x, y) d \tau .
\end{aligned}
$$

The boundary conditions will be satisfied if we let

$$
F(t, x, y)=\sum_{j, k=1}^{\infty} f_{j k}(t) \sin \frac{j \pi x}{a} \sin \frac{k \pi y}{b} .
$$

Here $f_{j k}(t)$ are unknown functions of time. Substituting (6) and (2) in (5), we obtain the ordinary integro-differential equation with variable coefficients (for the sake of simplicity $j k$ indices of $f_{j k}$ will be omitted later on):

$$
f^{\prime \prime}(t)+\left[\lambda^{2}+\mu \varphi(t)\right] f(t)=\varepsilon \lambda^{2} \int_{0}^{t} \Gamma(t-r) f(r) d r
$$

where

$$
\begin{aligned}
\lambda^{2} & =\Lambda^{2}\left(1-\frac{N_{10}}{N_{1 *}}-\frac{N_{20}}{N_{2 *}}\right), \\
\Lambda^{2} & =\frac{D}{m\left(n_{j}^{2}+m_{k}^{2}\right)^{2}}\left[\left(n_{j}^{2}+m_{k}^{2}\right)^{4}+\frac{12\left(1-v^{2}\right)}{h^{2}}\left(k_{2} n_{j}^{2}+k_{1} m_{k}^{2}\right)^{2}\right], \\
n_{j} & =\frac{j \pi}{a}, \quad m_{k}=\frac{k \pi}{b}, \\
\varepsilon \Gamma(t) & =\frac{D}{\lambda^{2} m\left(n_{j}^{2}+m_{k}^{2}\right)^{2}}\left[12\left(1-v^{2}\right) h^{-2}\left(k_{2} n_{j}^{2}+k_{1} m_{k}^{2}\right)^{2} \Gamma_{2}(t)+\left(n_{j}^{2}+m_{k}^{2}\right)^{4} \Gamma_{3}(t)\right], \\
\mu & =\frac{1}{m}\left(N_{11} n_{j}^{2}+N_{21} m_{k}^{2}\right), \quad N_{1 *}=m \Lambda^{2} n_{j}^{-2}, \quad N_{2 *}=m \Lambda^{2} m_{k}^{-2} .
\end{aligned}
$$

The viscous resistance of viscoelastic solid is much smaller then the elastic one. Using

$$
\Gamma_{2}(t)=-E^{\prime}(t) / E, \quad \Gamma_{3}(t)=-N^{\prime}(t) / N(0)
$$

we find

$$
\begin{aligned}
\varepsilon \int_{0}^{\infty} \Gamma(\tau) d \tau= & \left(1-\frac{\left(n_{j}^{2}+m_{k}^{2}\right)^{4} N_{\infty} / N(0)+12\left(1-v_{\infty}^{2}\right) h^{-2}\left(k_{2} n_{j}^{2}+k_{1} m_{k}^{2}\right) E_{\infty} / E}{\left(n_{j}^{2}+m_{k}^{2}\right)^{4}+12\left(1-v_{\infty}^{2}\right) h^{-2}\left(k_{2} n_{j}^{2}+k_{1} m_{k}^{2}\right)}\right) \\
& \times\left(1-\frac{N_{10}}{N_{1 *}}-\frac{N_{20}}{N_{2 *}}\right)^{-1} .
\end{aligned}
$$

If Poisson's ratio is constant, then

$$
\varepsilon \int_{0}^{\infty} \Gamma(\tau) d \tau=\left(1-\frac{G_{\infty}}{G}\right)\left(1-\frac{N_{10}}{N_{1 *}}-\frac{N_{20}}{N_{2 *}}\right)^{-1},
$$


where the sub index $\infty$ denotes the long-time moduli. For the viscoelastic solids $G_{\infty} / G<1$, $N_{\infty} / N<1$ and for viscoelastic fluid $G_{\infty}=0$.

Later on we will assume that the inequality

$$
\varepsilon \int_{0}^{\infty} \Gamma(\tau) d \tau<1
$$

is satisfied. We will serve the small positive parameter $\varepsilon$ as the separation parameter for different degrees of approximation terms.

\subsection{A circular cylindrical shell}

Assume that the circular cylindrical shell of radius $R$ and a thickness $h$ is loaded by a uniformly distributed radial load $q_{0}+q_{1} \varphi(t)$, and, in addition, compressed by a longitudinal force $P_{0}+P_{1} \varphi(t)$. We introduce a non-dimensional longitudinal coordinate $\alpha=z / R$, where $0 z$ is the axis of the cylinder, and the central angle $\beta$. If the effect of the tangential inertia forces and of the tangential components of the reduced loads is ignored, then the vibration of circular cylindrical elastic shells can be represented by a single equation (Bolotin 1964)

$$
\begin{gathered}
m R^{4} \frac{\partial^{2}}{\partial t^{2}} \nabla^{2} \nabla^{2} F+R^{2}\left[N_{1} \frac{\partial^{2}}{\partial \alpha^{2}}+N_{2}\left(\frac{\partial^{2}}{\partial \beta^{2}}+1\right)\right] \nabla^{2} \nabla^{2} F+D\left(\nabla^{2}+1\right)^{2} \nabla^{2} \nabla^{2} F \\
-\frac{G h^{3}}{6} \frac{\partial^{2}}{\partial \alpha^{2}}\left(\frac{\partial^{2}}{\partial \alpha^{2}}-\frac{\partial^{2}}{\partial \beta^{2}}\right) \nabla^{2} F+E h R^{2} \frac{\partial^{4} F}{\partial \alpha^{4}}=0,
\end{gathered}
$$

where

$$
\nabla^{2}=\frac{\partial^{2}}{\partial \alpha^{2}}+\frac{\partial^{2}}{\partial \beta^{2}}, \quad N_{1}=\frac{1}{2 \pi R}\left[P_{0}+P_{1} \varphi(t)\right], \quad N_{2}=R\left[q_{0}+q_{1} \varphi(t)\right] .
$$

As a boundary conditions, we will assume that at the edges of the shell $(z=0, z=l, l$ is the length of the shell) the radial displacement is zero.

Using the above mentioned approach we find the equation for the viscoelastic circular cylindrical shell

$$
\begin{aligned}
m R^{4} & \frac{\partial^{2}}{\partial t^{2}} \nabla^{2} \nabla^{2} F+R^{2}\left[N_{1} \frac{\partial^{2}}{\partial \alpha^{2}}+N_{2}\left(\frac{\partial^{2}}{\partial \beta^{2}}+1\right)\right] \nabla^{2} \nabla^{2} F+D\left(\nabla^{2}+1\right)^{2} \nabla^{2} \nabla^{2} F \\
& -\frac{G h^{3}}{6} \frac{\partial^{2}}{\partial \alpha^{2}}\left(\frac{\partial^{2}}{\partial \alpha^{2}}-\frac{\partial^{2}}{\partial \beta^{2}}\right) \nabla^{2} F+E h R^{2} \frac{\partial^{4} F}{\partial \alpha^{4}} \\
= & D \int_{0}^{t} \Gamma_{3}(t-\tau)\left(\nabla^{2}+1\right)^{2} \nabla^{2} \nabla^{2} F d \tau+\frac{G h^{3}}{6} \int_{0}^{t} \Gamma_{1}(t-\tau) \frac{\partial^{2}}{\partial \alpha^{2}}\left(\frac{\partial^{2}}{\partial \alpha^{2}}-\frac{\partial^{2}}{\partial \beta^{2}}\right) \nabla^{2} F d \tau \\
& +E h R^{2} \int_{0}^{t} \Gamma_{2}(t-\tau) \frac{\partial^{4} F}{\partial \alpha^{4}} d \tau
\end{aligned}
$$

We will seek the solution of (9) in the form

$$
F(t, \alpha, \beta)=\sum_{j, k=1}^{\infty} f_{j k}(t) \sin n \alpha \cos k \beta
$$


where $n=j \pi R / l$. Here $j$ denotes the number of half waves in the meridian direction, and $k$ gives the number of half waves in the circumferential direction. Substituting (10) in (9) and taking into account the expressions of $N_{1}, N_{2}$, we obtain ( $j k$ indices are omitted)

$$
f^{\prime \prime}(t)+\left[\lambda^{2}+\mu \varphi(t)\right] f(t)=\varepsilon \lambda^{2} \int_{0}^{t} \Gamma(t-r) f(r) d r
$$

where

$$
\begin{aligned}
\lambda^{2}= & \frac{D g(n, k)}{m R^{4}}\left(1-\frac{N_{10}}{N_{1 *}}-\frac{N_{20}}{N_{2 *}}\right), \quad \mu=\frac{1}{m R^{2}}\left[N_{11} n^{2}+N_{21}\left(k^{2}-1\right)\right] \\
g(n, k)= & \left(n^{2}+k^{2}\right)^{-2}\left[\left(n^{2}+k^{2}-1\right)^{2}\left(n^{2}+k^{2}\right)^{2}+(1-v) n^{2}\left(n^{4}-k^{4}\right)\right. \\
& \left.+12\left(1-v^{2}\right) n^{4} h^{-2} R^{2}\right], \\
\varepsilon \Gamma(t)= & \frac{1}{\lambda^{2} m R^{4}}\left[\frac{G h^{3} n^{2}}{6}\left(\frac{n^{2}-k^{2}}{n^{2}+k^{2}}\right) \Gamma_{1}(t)+\frac{E h R^{2} n^{4}}{\left(n^{2}+k^{2}\right)^{2}} \Gamma_{2}(t)+D\left(n^{2}+k^{2}-1\right)^{2} \Gamma_{3}(t)\right], \\
N_{10}= & \frac{P_{0}}{2 \pi R}, \quad N_{11}=\frac{P_{1}}{2 \pi R}, \quad N_{20}=R q_{0}, \quad N_{21}=R q_{1}, \\
N_{1 *}= & \frac{D g(n, k)}{n^{2} R^{2}}, \quad N_{2 *}=\frac{D g(n, k)}{\left(k^{2}-1\right) R^{2}} .
\end{aligned}
$$

Thus the problem is reduced to above obtained (7).

Example 1 Let us consider the simplest relaxation functions $G(t)=G e^{-q t}, K(t)=K e^{-r t}$, where $G, K, q$ and $r$ are positive constants. It is not difficult to obtain

$$
\begin{aligned}
\frac{8 \bar{G}(\bar{G}+3 \bar{K})}{3 \bar{K}+4 \bar{G}} & =2 G\left(e^{-q t}+\frac{1+v}{1-v} e^{-\sigma t}\right)=N(t), \\
v(t) & =v-\frac{2}{3}(1+v)(q-r) e^{-\left[r+\frac{2}{3}(1+v)(q-r)\right] t}
\end{aligned}
$$

where $\sigma=q+\frac{2(1-2 v)}{3(1-v)}(r-q)$. Using the derivative of $N(t)$ we find

$$
\Gamma_{3}(t)=\frac{1-v}{2} q e^{-q t}+\frac{1+v}{2} \sigma e^{-\sigma t} .
$$

By the same way we find

$$
\Gamma_{1}(t)=q e^{-q t}, \quad \Gamma_{2}(t)=\left[r+\frac{2}{3}(1+v)(q-r)\right] e^{-\left[r+\frac{2}{3}(1+v)(q-r)\right] t} .
$$

If $v(t)=v=$ const for $t>0$, then $\sigma=r=q$ and $\Gamma_{1}(t)=\Gamma_{2}(t)=\Gamma_{3}(t)=q e^{-q t}$, but if $\bar{K}=K=$ const for $t>0$, i.e. if $r=0$, then

$$
\Gamma_{1}(t)=q e^{-q t}, \quad \Gamma_{2}(t)=\frac{2}{3} q e^{-\frac{2}{3} q t}, \quad \Gamma_{3}(t)=\frac{1-v}{2} q e^{-q t}+\frac{(1+v)^{2}}{6(1-v)} q e^{-\frac{1+v}{3(1-v)} q t}
$$

are obtained. 


\section{The integro-differential Hill equation}

By means of various methods (separation of variables, finite differences with respect to space coordinates, Bubnov-Galerkin method, etc.) the problem of dynamic stability of viscoelastic plates, shells, columns etc. is reduced to the investigation of stability of zero solution of (7). Solution of the problem for any $\Gamma(t)$ is practically very important and difficult. We will reduce integro-differential equation (7) to a new integro-differential one, more explicitly describing the main mechanical properties of material and shells.

Using the Laplace transform, defined as Laplace-Carson integral without the factor $s$, from (7), for zero initial conditions, we get

$$
\bar{f}=\frac{-\mu \overline{(\varphi f)}}{s^{2}+\lambda^{2}-\varepsilon \lambda^{2} \bar{\Gamma}}
$$

where the line over the function denotes its Laplace transform with the complex parameter $s$. The function $\bar{f}$ represented by (12) and the image of kernel $\bar{\Gamma}$ are analytic in the right halfplane $\operatorname{Re} s>0$. We will suppose that these functions are analytically continued into the whole complex plane. In order to evaluate the inverse transform of (12) it is necessary to know the poles of this function which are the roots of the equation

$$
s^{2}+\lambda^{2}-\varepsilon \lambda^{2} \bar{\Gamma}=0
$$

Equation (13) is the frequency equation of free vibrations of viscoelastic shells.

Theorem 1 Let $\Gamma(t)$ be a positive monotonically decreasing convex and piecewise continuous function for $t \geq 0$, let it vanish for $t<0$ and let the inequality (8) be satisfied. (13) has just two complex conjugate roots having negative real parts and real negative roots.

Proof Let $-\alpha \pm i \beta$ be the roots of equation (13). Substituting $s=-\alpha+i \beta$ for (13) and splitting in real and imaginary parts, gives

$$
\begin{aligned}
& \hat{\Gamma}_{c} \equiv \int_{o}^{\infty} e^{\alpha \tau} \Gamma(\tau) \cos \beta \tau d \tau=\frac{\alpha^{2}+\lambda^{2}-\beta^{2}}{\varepsilon \lambda^{2}}, \\
& \hat{\Gamma}_{s} \equiv \int_{o}^{\infty} e^{\alpha \tau} \Gamma(\tau) \sin \beta \tau d \tau=\frac{2 \alpha \beta}{\varepsilon \lambda^{2}} .
\end{aligned}
$$

Thus (13) is equivalent to the system of two equations (14) for $\alpha$ and $\beta$. As we know the Fourier integrals $\hat{\Gamma}_{c}$ and $\hat{\Gamma}_{s}$ are convergent either the function $e^{\alpha t} \Gamma(t)$ satisfies the Dirichlet conditions for $0<t<\infty$, or the function is monotonically decreasing. Each of the functions $\hat{\Gamma}_{c}(\alpha, \beta)$ and $\hat{\Gamma}_{s}(\alpha, \beta)$ are continuous and tends to zero as $\beta$ tends to infinity through any set of values.

From (14) we find

$$
\begin{aligned}
\varepsilon \sqrt{\hat{\Gamma}_{c}^{2}+\hat{\Gamma}_{s}^{2}} & =\lambda^{-2}\left[\left(\alpha^{2}+\lambda^{2}-\beta^{2}\right)^{2}+4 \alpha^{2} \beta^{2}\right]^{1 / 2} \\
& =\frac{2 \varepsilon}{\lambda} \sqrt{\alpha^{2}+(\lambda-\beta)^{2}}\left(1-\frac{\lambda-\beta}{\lambda}+\frac{\alpha^{2}+(\lambda-\beta)^{2}}{4 \lambda^{2}}\right)^{1 / 2}
\end{aligned}
$$

and

$$
\varepsilon \sqrt{\hat{\Gamma}_{c}^{2}+\hat{\Gamma}_{s}^{2}}<1 .
$$


The inequalities $0<\varepsilon \hat{\Gamma}_{c}<1$ give $\beta^{2}>\alpha^{2}>0$. Thus $\alpha$ and $\beta$ are real and we may put $\beta>0$. As $\hat{\Gamma}_{s}>0$, from the second of the (14) we deduce $\alpha>0$.

The roots $\alpha$ and $\beta$ may be calculated by iteration procedure. From (14) we define the following system of iterations: $\alpha_{0}=0, \beta_{0}=\lambda$

$$
\begin{aligned}
\alpha_{n+1} & =\frac{\varepsilon \lambda^{2}}{2 \beta_{n}} \hat{\Gamma}_{s}\left(\alpha_{n}, \beta_{n}\right), \quad \beta_{n+1}=\left[\lambda^{2}+\alpha_{n+1}^{2}-\varepsilon \lambda^{2} \hat{\Gamma}_{c}\left(\alpha_{n+1}, \beta_{n}\right)\right]^{1 / 2}, \\
n & =0,1,2, \ldots
\end{aligned}
$$

Consider the integrals

$$
\hat{\Gamma}_{1 c}=\int_{o}^{\infty} \tau e^{\alpha \tau} \Gamma(\tau) \cos \beta \tau d \tau, \quad \hat{\Gamma}_{1 s}=\int_{o}^{\infty} \tau e^{\alpha \tau} \Gamma(\tau) \sin \beta \tau d \tau .
$$

Using the Dirichlet theorem we can prove the uniform convergences with respect to $\alpha$ and $\beta$ for $0 \leq \alpha \leq \varepsilon \Gamma(0) / 2, \lambda\left[1-\varepsilon \int_{0}^{\infty} \Gamma(\tau) d \tau\right]^{1 / 2} \leq \beta \leq \lambda$ of integrals $\hat{\Gamma}_{1 s}$ and $\hat{\Gamma}_{1 c}$. Therefore by the theorem on differentiation of an integral with respect to the parameter, the differentiation under the integral sign in (16) with respect to $\alpha$ and $\beta$ is valid. The derivatives of iteration vector-function with respect to $\alpha$ and $\beta$ are described by the integrals $\hat{\Gamma}_{1 s}$ and $\hat{\Gamma}_{1 c}$. To estimate these integrals, consider the product

$$
\begin{aligned}
2 \hat{\Gamma}_{s} \hat{\Gamma}_{c} & =2 \int_{0}^{\infty} \int_{0}^{\infty} e^{\alpha(\varsigma+\tau)} \Gamma(\varsigma) \Gamma(\tau) \sin \beta \varsigma \cos \beta \tau d \varsigma d \tau \\
& =\int_{0}^{\infty} \int_{0}^{\infty} \Omega(\varsigma, \tau) e^{\alpha(\varsigma+\tau)} \Gamma(\varsigma+\tau) \sin \beta(\varsigma+\tau) d \varsigma d \tau
\end{aligned}
$$

where $\Omega(\varsigma, \tau)=\Gamma(\varsigma) \Gamma(\tau) / \Gamma(\varsigma+\tau)$ is a monotonically decreasing convex function of two variables in the domain $X=\{(\varsigma, \tau): \varsigma \geq 0, \tau \geq 0\}$. Besides $\Omega(0, \tau)=\Omega(\varsigma, 0)=$ $\Omega(0,0)=\Gamma(0), \lim _{\zeta \rightarrow \infty, \tau \rightarrow \infty} \Omega(\varsigma, \tau)=\Omega_{0}>0$ (for the exponential kernel the function $\Omega$ is constant, $\Omega=\Gamma(0)$ ). By the second mean-value theorem of integral calculus there is a point $\left(\varsigma^{*}, \tau^{*}\right) \in X$ so that

$$
2 \hat{\Gamma}_{s} \hat{\Gamma}_{c}=\Omega\left(\varsigma^{*}, \tau^{*}\right) \int_{0}^{\infty} \int_{0}^{\infty} e^{\alpha(\varsigma+\tau)} \Gamma(\varsigma+\tau) \sin \beta(\varsigma+\tau) d \varsigma d \tau .
$$

Using functions

$$
\varsigma=\frac{s \cos \varphi}{\sqrt{2} \cos (\pi / 4-\varphi)}, \quad \tau=\frac{s \sin \varphi}{\sqrt{2} \cos (\pi / 4-\varphi)}, \quad 0 \leq s<+\infty, 0 \leq \varphi \leq \pi / 2,
$$

and taking $\varsigma+\tau=s$ into account, after changing variables in the double integral, we find $2 \hat{\Gamma}_{s} \hat{\Gamma}_{c}=\Omega\left(\varsigma^{*}, \tau^{*}\right) \hat{\Gamma}_{1 s}$. By the same way $\hat{\Gamma}_{c}^{2}-\hat{\Gamma}_{s}^{2}=\Omega\left(\varsigma^{* *}, \tau^{* *}\right) \hat{\Gamma}_{1 c}$ is obtained, here $\left(\varsigma^{* *}, \tau^{* *}\right) \in X$. The last two relations together with inequality (15) secure the convergence of iteration (14) to a unique limit.

Example 2 The kernel of constitutive equation for the Kelvin material is written as $\varepsilon \Gamma(t)=$ $-\frac{\eta}{E} \dot{\delta}(t)$, where $\delta(t)$ is Dirac's delta. It is easy to get $\varepsilon \Gamma_{s}=\eta \lambda / E, \varepsilon \Gamma_{c}=0$. From (14) we obtain

$$
\alpha=\frac{\eta \lambda^{2}}{2 E}, \quad \beta^{2}=\lambda^{2}-\frac{\eta^{2} \lambda^{4}}{4 E^{2}}
$$


For sufficiently small $\varepsilon$ we find the following solutions from (16)

$$
\alpha=\frac{\varepsilon \lambda \Gamma_{s}}{2}+\varepsilon^{2} \lambda \omega_{1}+\varepsilon^{3} \theta_{1}+\cdots, \quad \beta=\lambda-\gamma, \quad \gamma=\frac{\varepsilon \lambda \Gamma_{c}}{2}+\varepsilon^{2} \lambda \omega_{2}+\varepsilon^{3} \theta_{2}+\cdots,
$$

where

$$
\begin{aligned}
\omega_{1} & =\frac{1}{4}\left(\Gamma_{s} \Gamma_{c}-\lambda \Gamma_{c} \Gamma_{1 c}+\lambda \Gamma_{s} \Gamma_{1 s}\right), \\
\omega_{2} & =\frac{1}{8}\left(\Gamma_{c}^{2}-\Gamma_{s}^{2}+2 \lambda \Gamma_{s} \Gamma_{1 c}+2 \lambda \Gamma_{c} \Gamma_{1 s}\right), \\
\theta_{1} & =\frac{\lambda}{2}\left[\Gamma_{s} \omega_{2}+\Gamma_{c} \omega_{1}-\lambda \omega_{2} \Gamma_{1 c}+\lambda \omega_{1} \Gamma_{1 s}-\frac{\lambda^{2}}{4} \Gamma_{s} \Gamma_{c} \Gamma_{2 c}+\frac{\lambda^{2}}{8} \Gamma_{2 s}\left(\Gamma_{s}^{2}-\Gamma_{c}^{2}\right)\right], \\
\theta_{2} & =\frac{\lambda}{2}\left[\Gamma_{c} \omega_{2}-\Gamma_{s} \omega_{1}+\lambda \omega_{1} \Gamma_{1 c}+\lambda \omega_{2} \Gamma_{1 s}+\frac{\lambda^{2}}{4} \Gamma_{s} \Gamma_{c} \Gamma_{2 s}+\frac{\lambda^{2}}{8} \Gamma_{2 s}\left(\Gamma_{s}^{2}-\Gamma_{c}^{2}\right)\right], \\
\Gamma_{k c} & =\int_{0}^{\infty} t^{k} \Gamma(t) \cos \lambda t d t, \quad \Gamma_{k s}=\int_{0}^{\infty} t^{k} \Gamma(t) \sin \lambda t d t, \quad k=0,1,2
\end{aligned}
$$

and $\Gamma_{0 c}=\Gamma_{c}, \Gamma_{0 s}=\Gamma_{s}$ denote the cos- and sin- Fourier transforms of $\Gamma(t)$, respectively. From (14) we deduce the known in literature results

$$
\begin{aligned}
\lim _{\lambda \rightarrow \infty} \frac{\beta}{\lambda} & =1, \quad \lim _{\lambda \rightarrow \infty} \alpha=\frac{\varepsilon \Gamma(0)}{2} ; \\
\beta^{2} & \approx \lambda^{2}\left[1-\varepsilon \int_{0}^{\infty} \Gamma(\tau) d \tau\right]=\lambda^{2} E_{\infty} / E_{0}, \quad \alpha \approx 0 \quad \text { for } \lambda \ll 1 .
\end{aligned}
$$

As we see for $\lambda \gg 1$, the frequencies of elastic and viscoelastic vibrations coincide and the damped coefficient has its maximum value. If the creep is restricted, i.e. the long-time modulus $E_{\infty}>0$, the limiting state of a viscoelastic body as $t \rightarrow \infty$ is described by the equations of the theory of elasticity with long-time moduli.

Let us rewrite (12) in the form

$$
\bar{f}\left[(s+\alpha)^{2}+\beta^{2}\right]+\mu \overline{(\varphi f)}+\mu \frac{\bar{B}(s) \overline{(\varphi f)}}{1-\bar{B}(s)}=0
$$

where

$$
\bar{B}(s)=\frac{\varepsilon \lambda^{2} \bar{\Gamma}+2 \alpha s+\alpha^{2}+\beta^{2}-\lambda^{2}}{(s+\alpha)^{2}+\beta^{2}}=\frac{2 \alpha(s+\alpha)+\varepsilon \lambda^{2}\left(\bar{\Gamma}-\hat{\Gamma}_{c}\right)}{(s+\alpha)^{2}+\beta^{2}} .
$$

Here we find

$$
B(0)=\lim _{s \rightarrow \infty} s \bar{B}(s)=2 \alpha, \quad B(\infty)=\lim _{s \rightarrow 0} s \bar{B}(s)=0 .
$$

The numbers $-\alpha \pm i \beta$ are the zeros of divisor, indeed

$$
D(-\alpha \pm i \beta)=2 \alpha( \pm i \beta) \mp i \varepsilon \lambda^{2} \hat{\Gamma}_{s}=i\left( \pm 2 \alpha \beta \mp \varepsilon \lambda^{2} \hat{\Gamma}_{s}\right),
$$

which is equal to zero according to (14). By using L'Hospital's rule, the limits

$$
\lim _{s \rightarrow-\alpha \pm i \beta} \bar{B}(s)=\lim _{s \rightarrow-\alpha \pm i \beta} \frac{2 \alpha+\left.\varepsilon \lambda^{2} \frac{d}{d s} \hat{\Gamma}(s)\right|_{s=-\alpha \pm i \beta}}{2(s+\alpha)}=\frac{2 \alpha-\varepsilon \lambda^{2}\left(\hat{\Gamma}_{1 c} \mp i \hat{\Gamma}_{1 s}\right)}{ \pm 2 i \beta}
$$

are obtained. Thus the numbers $-\alpha \pm i \beta$ are not the poles of the function $\bar{B}(s)$. 
Using (14) we find the following inverse transformation of the function

$$
B(t)=\frac{\varepsilon \lambda^{2}}{\beta} e^{-\alpha t} \int_{t}^{\infty} \Gamma(\tau) e^{\alpha \tau} \sin \beta(\tau-t) d \tau .
$$

This is a monotonically decreasing continuous function having series expression

$$
B(t)=e^{-\alpha t}\left[2 \alpha-\varepsilon \lambda^{2} \Gamma_{c} t+\frac{t^{2}}{2} \varepsilon \lambda^{2}\left(\Gamma_{0}-\beta \hat{\Gamma}_{s}\right)+\cdots\right],
$$

where for the regular kernel $\Gamma_{0}=\Gamma(0)$. It is seen that $B(t) \leq 2 \alpha e^{-\alpha t}$ for $t \geq 0$. Representing

$$
B(t)=\frac{\varepsilon \lambda^{2}}{\beta} \int_{0}^{\infty} \Gamma(t+\tau) e^{\alpha \tau} \sin \beta \tau d \tau=\Gamma(t) \frac{\varepsilon \lambda^{2}}{\beta} \int_{0}^{\infty} \frac{\Gamma(t+\tau)}{\Gamma(t)} e^{\alpha \tau} \sin \beta \tau d \tau,
$$

we see that $B(t)=O(\Gamma(t))$ for $t \gg 1$.

Let us denote

$$
\bar{\Phi}(s)=\frac{1}{2 \alpha} \frac{\bar{B}(s)}{1-\bar{B}(s)}=\frac{s+\alpha+\frac{\varepsilon \lambda^{2}}{2 \alpha}\left(\bar{\Gamma}-\hat{\Gamma}_{c}\right)}{s^{2}+\lambda^{2}(1-\varepsilon \bar{\Gamma})} .
$$

Theorem 2 Let $\Gamma(t)$ satisfies the conditions of Theorem 1 . The numbers $-\alpha \pm i \beta$ are not the poles of the function $\bar{\Phi}(s)$. Moreover, the function $\Phi(t)$ is positive, continuous, monotonically decreasing so that $\Phi(0)=1, \Phi(\infty)=0$ and have the series expansion

$$
\Phi(t)=e^{-\alpha t}\left[1-t\left(\frac{\varepsilon \lambda^{2}}{2 \alpha} \hat{\Gamma}_{c}-2 \alpha\right)+t^{2} \varepsilon \lambda^{2}\left(\frac{\Gamma_{0}-\beta \hat{\Gamma}_{s}}{4 \alpha}-\hat{\Gamma}_{c}\right)+\cdots\right]
$$

Proof From the expression of $\bar{\Phi}(s)$ the limits $\Phi(0)=\lim _{s \rightarrow \infty} s \bar{\Phi}(s)=1, \Phi(\infty)=$ $\lim _{s \rightarrow 0} s \bar{\Phi}(s)=0$ are obtained. Using L'Hospital's rule we find

$$
\lim _{s \rightarrow-\alpha \pm i \beta} \bar{\Phi}(s)=\frac{1-\frac{\varepsilon \lambda^{2}}{2 \alpha}\left(\hat{\Gamma}_{1 c} \mp i \hat{\Gamma}_{1 s}\right)}{-2 \alpha \pm 2 i \beta+\varepsilon \lambda^{2}\left(\hat{\Gamma}_{1 c} \mp i \hat{\Gamma}_{1 s}\right)} .
$$

Thus $-\alpha \pm i \beta$ are not the poles of the function $\bar{\Phi}(s)$. As $|\bar{B}(s)|<1$ for $\operatorname{Re} s>\operatorname{Re} s_{0}>0$, we have the absolutely and uniformly convergent series

$$
\bar{\Phi}(s)=\frac{1}{2 \alpha}\left[\bar{B}(s)+\bar{B}^{2}(s)+\cdots\right] .
$$

Here the original

$$
\Phi(t)=\frac{1}{2 \alpha} B(t)+\frac{1}{2 \alpha} \int_{0}^{t} B(t-\tau) B(\tau) d \tau+\cdots
$$

and the series expansion (21) are obtained. Note that the second term is proportional to the parameter $\alpha$. Using $B(t) \leq 2 \alpha e^{-\alpha t}$ and the convolution of functions we find

$$
B_{n}(t) \leq 2 \alpha \frac{(2 \alpha t)^{n-1}}{(n-1) !} e^{-\alpha t}, \quad n=1,2, \ldots
$$




$$
B_{n}(t)=\int_{0}^{t} B_{n-1}(t-\tau) B_{1}(\tau) d \tau, \quad B_{1}(t)=B(t)
$$

Then

$$
|\Phi(t)| \leq \sum_{n=1}^{\infty} \frac{(2 \alpha t)^{n-1}}{(n-1) !} e^{-\alpha t}=e^{\alpha t},
$$

thus the series for $\Phi(t)$ is absolutely convergent for any finite $t$.

Example 3 For the kernel $\Gamma(t)=\sum_{k=1}^{N} q_{k} e^{-\eta_{k} t}\left(0<\eta_{1}<\cdots<\eta_{N}\right)$ it is not difficult to find $B(t)=\sum_{k=1}^{N} \varepsilon c_{k} e^{-\eta_{k} t}, \Phi(t)=\sum_{k=1}^{N} \chi_{k} e^{-\rho_{k} t}$, where the real negative numbers $-\rho_{k} \approx$ $-\eta_{k}+\varepsilon c_{k}(k=1,2, \ldots, N)$ are the roots of the equation $1-\bar{B}(s)=0$, so that

$$
\begin{aligned}
c_{k} & =\lambda^{2} q_{k} /\left[\beta^{2}+\left(\eta_{k}-\alpha\right)^{2}\right], \\
\chi_{k} & =\frac{1}{2 \alpha}\left(\sum_{i=1}^{N} \frac{\varepsilon c_{i}}{\eta_{i}-\rho_{k}}\right)\left[\sum_{j=1}^{N} \frac{c_{j} \varepsilon}{\left(\eta_{j}-\rho_{k}\right)^{2}}\right]^{-1}, \quad \sum_{k=1}^{N} \varepsilon c_{k}=2 \alpha .
\end{aligned}
$$

Moreover, $B(t)<\sum_{k=1}^{N} \varepsilon c_{k} e^{-\eta_{1} t}=2 \alpha e^{-\eta_{1} t}<2 \alpha e^{-\alpha t}$ for $t>0$. For $N=1$ we have $B(t)=$ $2 \alpha e^{-\eta t}, \Phi(t)=e^{-(\eta-2 \alpha) t}, 2 \alpha=\varepsilon \lambda^{2} q\left[\beta^{2}+(\eta-\alpha)^{2}\right]^{-1}$. From (8) we find $\eta>\varepsilon q$. As the product of the roots $-\alpha \pm i \beta$ and $-\eta+2 \alpha$ are equal to $-\lambda^{2}(\eta-\varepsilon q)$, that is $\left(\alpha^{2}+\beta^{2}\right)(-\eta+$ $2 \alpha)=-\lambda^{2}(\eta-\varepsilon q)$, then $\eta>2 \alpha$ is obtained.

From (20) we get the following integro-differential equation

$$
f^{\prime \prime}+2 \alpha f^{\prime}+\left[\omega^{2}+\mu \varphi(t)\right] f+2 \alpha \mu \int_{0}^{t} \Phi(t-\tau) \varphi(\tau) f(\tau) d \tau=0
$$

where $\omega^{2}=\alpha^{2}+\beta^{2}$. If we neglect the integral term, Hill's equation will be obtained. This term is proportional to the product of the two small parameters $\alpha$ and $\mu$. Thus the problem of dynamic stability analysis of viscoelastic shells is reduced to the determination of the stability criteria for the zero solution of (22). In the problems of dynamic stability of elastic systems with damping $\alpha, \omega, \mu$ are independent parameters (Bolotin 1964). But for viscoelastic vibrations $\alpha$ and $\beta$ are defined from (16) or (17) and $\omega^{2}=\alpha^{2}+\beta^{2}, \beta=\lambda-\gamma$, with $\lambda$ the frequency of elastic vibrations. If viscosity of material is absent then $\alpha=\gamma=0$ and $\omega=\beta=\lambda$.

Theorem 3 Equations (7) and (22) are equivalent, i.e. the solution of (7) for some initial conditions is the solution of (22) for the same initial conditions and vice versa.

Proof Let the function $f(t)$ be the solution of (7) for some initial conditions. We will show that this function returns (22) into identity. Subtracting identity (7) for the function $f(t)$ from (22) we get the equation

$$
2 \alpha f^{\prime}+\left(\omega^{2}-\lambda^{2}\right) f+2 \alpha \mu \int_{0}^{t}\left[2 \alpha \mu \Phi(t-\tau) \varphi(\tau)+\varepsilon \lambda^{2} \Gamma(t-\tau)\right] f(\tau) d \tau=0
$$

with zero initial conditions. Using the Laplace transform we obtain

$$
\left(2 \alpha s+\beta^{2}+\alpha^{2}-\lambda^{2}+\varepsilon \lambda^{2} \bar{\Gamma}\right) \bar{f}+2 \alpha \mu \bar{\Phi} \overline{(\varphi f)}=0 .
$$


Substituting the expression for $\bar{\Phi}$ gives

$$
\left[s^{2}+\lambda^{2}(1-\varepsilon \bar{\Gamma})\right] \bar{f}+\mu \overline{(\varphi f)}=0 .
$$

This is the Laplace transform of the identity (7) with zero initial conditions.

\section{The regions of dynamic instability and parametric resonance}

Equation (22) contains three parameters $\alpha, \omega, \mu$ and the function $\Phi(t)$. Finding the stability and instability domains in the parameter space is the main problem for the stability theory. We will investigate how the stable vibrations of shells become unstable or vice versa changing these parameters.

Using $f=x_{1}, \dot{f}=x_{2}$ we reduce (22) to the system of linear differential equations

$$
\dot{x}_{1}=x_{2}, \dot{x}_{2}=-\left[\omega^{2}+\mu \varphi(t)\right] x_{1}-2 \alpha \mu \int_{0}^{t} \Phi(t-\tau) \varphi(\tau) x_{1}(\tau) d \tau-2 \alpha x_{2} .
$$

Matrix of this system is represented as

$$
\begin{aligned}
A(t) & =A_{0}+\mu \varphi A_{1}+2 \mu \alpha \int_{0}^{t} \Phi(t-\tau) \varphi(\tau) A_{1}(\mathrm{o}) d \tau, \\
A_{0} & =\left(\begin{array}{lc}
0 & 1 \\
-\omega^{2} & -2 \alpha
\end{array}\right), \quad A_{1}=\left(\begin{array}{ll}
0 & 0 \\
-1 & 0
\end{array}\right)
\end{aligned}
$$

where the sign (o) denotes the place of multiplied function of variable $\tau$. The solution of the problem $\dot{x}=A(t) x, x(0)=x_{0}$ is written as $x(t)=U(t) x_{0}$, where the evolution matrix $U(t)$ is the solution of the problem

$$
\dot{U}=A(t) U, \quad U(0)=I .
$$

If $\omega>\alpha$ the solution of this problem for the matrix $A_{0}$ is

$$
U_{0}(t)=e^{A_{0} t}=e^{-\alpha t}\left(\begin{array}{cc}
\cos \beta t+\frac{\alpha}{\beta} \sin \beta t & \frac{1}{\beta} \sin \beta t \\
-\frac{\omega^{2}}{\beta} \sin \beta t & \cos \beta t-\frac{\alpha}{\beta} \sin \beta t
\end{array}\right)
$$

where $\beta=\sqrt{\omega^{2}-\alpha^{2}}$ is the frequency of damped vibrations and $\alpha$ is a damping factor. If $\omega=\alpha$, the matrix $A_{0}$ has two equal eigenvalues $\omega$ and the vibration does not take place. If $\omega<\alpha$, the eigenvalues of matrix $A_{0}$ are real and negative and the vibration does not yet happen. For last two cases the solutions, corresponding to matrix $A_{0}$, are stable. Further only the first case will be taken into account.

Let $U(t)=U_{0}(t) V(t)$, then the problem (24) is reduced to

$$
\dot{V}=\mu \varphi U_{0}^{-1} A_{1} U_{0} V+2 \mu \alpha \int_{0}^{t} \Phi(t-\tau) \varphi(\tau) U_{0}^{-1}(t) A_{1} U_{0}(\tau) V(\tau) d \tau, \quad V(0)=I .
$$

If the matrices $U_{0}^{-1}$ and $U_{0}$ depend on the same argument $t$, then

$$
U_{0}^{-1} A_{1} U_{0}=\left(\begin{array}{cc}
\frac{1}{2 \beta} \sin 2 \beta t+\frac{\alpha}{2 \beta^{2}}(1-\cos 2 \beta t) & \frac{1}{2 \beta^{2}}(1-\cos 2 \beta t) \\
-\frac{\omega^{2}}{2 \beta^{2}}-\frac{\beta^{2}-\alpha^{2}}{2 \beta^{2}} \cos 2 \beta t-\frac{\alpha}{\beta} \sin 2 \beta t & -\frac{1}{2 \beta} \sin 2 \beta t-\frac{\alpha}{2 \beta^{2}}(1-\cos 2 \beta t)
\end{array}\right) .
$$


Assuming $\mu$ is sufficiently small, we see that system (25) has a standard form and its solution may be obtained by the averaging method. After averaging we find

$$
\frac{1}{T} \int_{0}^{T} \varphi U_{0}^{-1} A_{1} U_{0} d t=\left(\begin{array}{cc}
a-\frac{\alpha(b-c)}{\beta} & \frac{c-b}{\beta} \\
\frac{\alpha^{2}-\beta^{2}}{\beta} b-2 \alpha a-\frac{\omega^{2} c}{\beta} & -a+\frac{\alpha(b-c)}{\beta}
\end{array}\right) \equiv C
$$

where

$$
\begin{aligned}
& a=\frac{1}{2 \beta T} \int_{0}^{T} \varphi(\tau) \sin 2 \beta \tau d \tau, \quad b=\frac{1}{2 \beta T} \int_{0}^{T} \varphi(\tau) \cos 2 \beta \tau d \tau \\
& c=\frac{1}{2 \beta T} \int_{0}^{T} \varphi(t) d t .
\end{aligned}
$$

Let us average the last term in (25). Remembering that $\Phi(t) \leq e^{-\alpha t}$ and using the main-value theorem for integral in the point $\tau \in[0, T]$ we get

$$
\frac{1}{T} \int_{0}^{T} \int_{0}^{t} \Phi(t-\tau) U_{0}^{-1}(t) \varphi U_{0}^{-1} A_{1} U_{0} d \tau d t \approx \frac{1}{2} \int_{0}^{T} \varphi(\tau) U_{0}^{-1}(\tau) A_{1} U_{0}(\tau) d \tau=\frac{T}{2} C .
$$

Averaging problem (25) is written as follows

$$
\dot{V}=\mu(1+\alpha T) C V, \quad V(0)=I .
$$

The problem has the solution $V(t)=\exp (\bar{\mu} C t)$ where $\bar{\mu}=\mu(1+\alpha T)$. It is easy to verify

$$
C^{2}=\left(a^{2}+b^{2}-c^{2}\right) I .
$$

Denoting $r=\sqrt{a^{2}+b^{2}-c^{2}}$, we find $C^{2 k}=r^{2 k} I, C^{2 k+1}=r^{2 k} C, k=1,2, \ldots$ and

$$
\begin{aligned}
V(t) & =I+\bar{\mu} t C+\frac{1}{2 !} \bar{\mu}^{2} t^{2} C^{2}+\frac{1}{3 !} \bar{\mu}^{3} t^{3} C^{3}+\cdots \\
& =I\left(1+\frac{1}{2 !} \bar{\mu}^{2} t^{2} r^{2}+\frac{1}{4 !} \bar{\mu}^{4} t^{4} r^{4}+\cdots\right)+C\left(\bar{\mu} t+\frac{1}{3 !} \bar{\mu}^{3} t^{3} r^{2}+\cdots\right) \\
& =I \cosh (\bar{\mu} t r)+\frac{C}{r} \sinh (\bar{\mu} t r) .
\end{aligned}
$$

Approximate solution of problem (24) is written as

$$
U(t)=U_{0}(t) \cosh (\bar{\mu} r t)+U_{0}(t) C \frac{1}{r} \sinh (\bar{\mu} r t) .
$$

This is the approximate value of fundamental matrix of system (23). As we see this approach does not change the frequency of vibrations, but the damping factor and the character of the solution have been changed: if $\mu \leq \alpha / r(1+\alpha T)$ the solution is stable and if $\mu>\alpha / r(1+$ $\alpha T)$ it is instable. It is seen that the effect of the last term in (25) to the results may be essential.

If we put $t=T$ the monodromy matrix is obtained from (26). Eigenvalues of monodromy matrix $U(T)$ are the roots of the equation $\operatorname{det}[U(T)-\rho I]=0$, which may be written as

$$
\rho^{2}-\rho \operatorname{Tr} U(T)+\operatorname{det} U(T)=0 .
$$


Since

$$
\operatorname{det} U(T)=\exp \left(\int_{0}^{T} \operatorname{Tr} A d \tau\right)=\exp (-2 \alpha T)
$$

obtained exactly, we use monodromy matrix to find its trace $\operatorname{Tr} U(T)$. Solve for the eigenvalue $\rho$ via the quadratic formula

$$
\rho_{1,2}=\frac{\operatorname{Tr} U \pm \sqrt{(\operatorname{Tr} U)^{2}-4 \operatorname{det} U}}{2} .
$$

The sign of the discriminant $\Delta=(\operatorname{Tr} U)^{2}-4 \operatorname{det} U$ determines whether we have two distinct real eigenvalues, one repeated real eigenvalue or a complex conjugate pair of eigenvalues. The condition $\Delta=(\operatorname{Tr} U)^{2}-4 \operatorname{det} U=0$ gives us two equal roots, of which $\left|\rho_{1}\right|=\left|\rho_{2}\right|=\exp (-\alpha T)$. Since $x(t+n T)=\rho x(t+(n-1) T)=\cdots=\rho^{n} x(t)$ and the state $t \rightarrow \infty$ is equivalent to $n \rightarrow \infty$, then if $|\rho|=1$ the zero solution $x \equiv 0$ is stable, if $|\rho|<1$ is asymptotic stable, and if $|\rho|>1$ is instable. If $\rho=1$ the solution is $T$-periodical. For $\alpha>0$ we see that $\left|\rho_{i}<1\right|$, therefore the zero solution of system (23) is asymptotic stable, if $\alpha=0$ it is stable and if $\alpha<0$ it is instable.

It is not difficult to verify

$$
\operatorname{Tr} U(T)=2 e^{-\alpha T} \cosh (\bar{\mu} r T) \cos \beta T-\frac{2 c}{r} e^{-\alpha T} \sinh (\bar{\mu} r T) \sin \beta T .
$$

So (27) is written as

$$
\rho^{2}-2 \rho e^{-\alpha T}\left[\cosh (\bar{\mu} r T) \cos \beta T-\frac{c}{r} \sinh (\bar{\mu} r T) \sin \beta T\right]+e^{-2 \alpha T}=0
$$

and has the solutions

$$
\begin{aligned}
\rho_{1,2}= & e^{-\alpha T}\left\{\cosh (\bar{\mu} r T) \cos \beta T-\frac{c}{r} \sinh (\bar{\mu} r T) \sin \beta T\right. \\
& \pm \sqrt{\left.\left[\cosh (\bar{\mu} r T) \cos \beta T-\frac{c}{r} \sinh (\bar{\mu} r T) \sin \beta T\right]^{2}-1\right\} .}
\end{aligned}
$$

If $\mu=0$ we get

$$
\rho_{1,2}=e^{-\alpha T}(\cos \beta T \pm i \sin \beta T)
$$

For the values $\beta T \neq k \pi, k=1,2, \ldots$ multiplicators are complex-conjugate and $\left|\rho_{1}\right|=$ $\left|\rho_{2}\right|=\exp (-\alpha T)$. For the values $\beta T=k \pi, k=1,2, \ldots$ multiplicators are real and one of them may be greater than unity. In this case from (29) for $\left|\rho_{1}\right|>1$ we find

$$
\left|\cosh (\bar{\mu} r T) \cos \beta T-\frac{c}{r} \sinh (\bar{\mu} r T) \sin \beta T\right|>\cosh \alpha T .
$$

This inequality defines the regions of instability of the zero solution of (22). Note that the condition $\Delta=(\operatorname{Tr} U)^{2}-4 \operatorname{det} U>0$, which corresponds to existence of two distinct real eigenvalues, gives us the inequality like (30), for which the right hand side is equal to one. Such condition is not sufficient for appearance instability of solution for $\alpha>0$. It is obvious that for appearance of instability the amplitude of periodic load should satisfy the inequality 
$\mu>\alpha / r(1+\alpha T)$. As we see the last term in (22) reduces to decreasing the amplitude of external force required to cause dynamic instability of the plate. The inequality

$$
\frac{d}{d \alpha}\left(\frac{\alpha}{1+\alpha T}\right)=\frac{1}{(1+\alpha T)^{2}}>0
$$

shows that the greater the viscous strength of material of the plate (with the same values of other parameters), the greater the amplitude of force required to cause dynamic instability of the shell.

Theorem 4 In the parameter space $(\alpha, \mu, \omega)$ inequality (30) defines the half-conical domains of instability of the solution of (22).

Proof Since the damping factor $\alpha$ and the amplitude of external force $\mu$ are usually very small in comparison with the unit, we can simplify formula (30) by neglecting the terms containing higher powers of $\alpha$ and $\mu$. For $\alpha=0, \mu=0$ considering $\beta \rightarrow \omega$, from (30) we get $|\cos \omega T| \geq 1$, i.e. $\omega T=k \pi, k=1,2, \ldots$ Expanding inequality (30) the series around the values

$$
\alpha=0, \quad \mu=0, \quad \omega=\frac{k \pi}{T} \quad(k=1,2, \ldots)
$$

up to $\mu^{2}$ we will get

$$
r_{k}^{2} \bar{\mu}^{2} \geq \alpha^{2}+\left(\omega-\frac{k \pi}{T}-\gamma+\bar{\mu} c_{k}\right)^{2}, \quad \alpha \geq 0
$$

where $r_{k}^{2}=a_{k}^{2}+b_{k}^{2}$,

$$
\begin{aligned}
a_{k} & =\frac{1}{2 k \pi} \int_{0}^{T} \varphi(r) \sin \frac{2 k \pi r}{T} d r, \quad b_{k}=\frac{1}{2 k \pi} \int_{0}^{T} \varphi(r) \cos \frac{2 k \pi r}{T} d r, \\
c_{k} & =\frac{1}{2 k \pi} \int_{0}^{T} \varphi(r) d r .
\end{aligned}
$$

The region of instability corresponding to $k=1$ is called the principal region of dynamic instability. For $k=1,3,5, \ldots$ the period of solution is $2 T$, but for $k=2,4,6, \ldots$ the period is equal to $T$. As it is seen two solutions of identical periods bound the region of instability and two solutions of different periods bound the region of stability (Fig. 1). The parameter space is divided into stability and instability domains.

In the space of parameters $(\alpha, \mu, \omega)$ condition (31) defines the half conical domains with the axis $\omega=\frac{k \pi}{T}+\mu c_{k}$ and the generators $\omega=\frac{k \pi}{T}+\mu c_{k} \pm \mu r_{k}$ on the plane $\alpha=0$, as far as $\alpha=0$ implies $\varepsilon=0$ and $\gamma=0$. Since $r_{k}$ decreases for increasing $k$, the angle between these lines becomes narrower. The intersections of conies (31) with the plane $\alpha=$ const $>0$ are the following hyperbolas

$$
\frac{(1+\alpha T)^{2} r_{k}^{2}}{\alpha^{2}} \mu^{2}-\left(\frac{\omega-\frac{k \pi}{T}-\gamma+\mu(1+\alpha T) c_{k}}{\alpha}\right)^{2} \geq 1 .
$$

Here the lower bound $\mu_{\min } \geq \frac{\alpha}{r_{k}(1+\alpha T)}$ for the amplitude of external force is found. For increasing $k$ the fraction $\frac{\alpha}{r_{k}(1+\alpha T)}$ also increases, therefore the corresponding domains of instability is far away from the $\omega$ axis. Hyperbola (32) does not intersect with the $\omega$ axis, its 
Fig. 1 The domains of instability

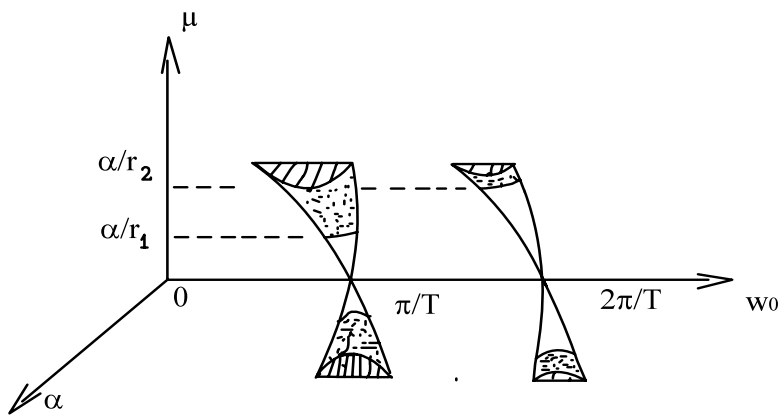

real semi-axis equals to $\alpha / r_{k}(1+\alpha T)$, the vertex is situated on the point $\mu=\alpha / r_{k}(1+\alpha T)$ and the asymptotes are found from the inequalities

$$
-c_{k}-r_{k}<\frac{\omega-k \pi / T}{\mu}<r_{k}-c_{k}, \quad \alpha=0
$$

i.e. for $\alpha=0$ the resonance domains begin from the points $\omega=\frac{k \pi}{T}$. We see that the width of the region of dynamic instability rapidly decreases as the number of the region increases. The numbers $\mu c_{k}$ indicate the differences between the resonance frequencies and the numbers $k \pi / T$.

The bound of domain (33) are defined by the equations

$$
\mu=\frac{1}{r_{k}-c_{k}}\left(\omega-\frac{k \pi}{T}\right), \quad \mu=\frac{-1}{r_{k}+c_{k}}\left(\omega-\frac{k \pi}{T}\right) .
$$

Tangent of the doubled angle between the axis of the cone and the vertical line to the $\omega$ axis is equal to $2 c_{k} /\left(1+r_{k}^{2}-c_{k}^{2}\right)$. For $c_{k}=0$, i.e. if the middle value of the periodic actions is zero, the axis of the cone is perpendicular to the $\omega$ axis. For increasing $k$ the numbers $c_{k}$ and $r_{k}$ are decreasing, therefore the axis of resonance cones becomes perpendicular to $\omega$ axis. With increasing viscosity the parameter $\gamma$ also increases and the vertex of the cone retires to the right-hand side of the plane $\omega=k \pi / T$ as $\gamma$ and to the left-hand side as $\mu c_{k}$.

Cross-section of half-cone (31) by the plane $\mu=$ const provides half-circle with the radius $\bar{\mu} r_{k}$ and the center is on the point $\alpha=0, \omega=\frac{k \pi}{T}-\mu c_{k}$. While increasing $\alpha$ the instability domains along $\omega$ axis becomes narrower and vanishes for $\alpha /(1+\alpha T)=\mu r_{k}$ at all. This means that the motion cannot be unstable if the damping is so high that the inequality $\alpha /(1+\alpha T)>\mu r_{k}$ holds. Since for increasing $k$ the numbers $a_{k}, b_{k}, c_{k}, r_{k}$ decrease, cones (31) also become narrower with increasing the number of resonance domains. In this case the amplitude of external perturbation $\mu_{\min }=\frac{\alpha}{r_{k}(1+\alpha T)}$ also increases.

Example 4 If $T$-periodic function $\varphi(t)$ in the domain $0<t<T$ is given as Dirac's delta $\varphi(t)=\delta\left(t-\frac{T}{2}\right)$, we find $a_{k}=0, b_{k}=\frac{(-1)^{k}}{2 k \pi}, c_{k}=r_{k}=\frac{1}{2 k \pi}$. Inequality (31) becomes

$$
\mu^{2} \geq\left(\frac{2 k \pi}{T(1+\alpha T)}\right)^{2}\left[\alpha^{2} T^{2}+\left(\omega T-k \pi-\gamma T+\frac{\mu(1+\alpha T) T}{2 k \pi}\right)^{2}\right] .
$$

For the parametric resonance could take place, the amplitudes of the external actions should be greater than the certain positive numbers for the above mentioned formula. Moreover, the lower bound increases while the numbers of instability regions increase. 
Example 5 For the $T$-periodic function

$$
\varphi(t)=\left\{\begin{aligned}
p, & 0 \leq t \leq T / 2 \\
-p, & T / 2 \leq t \leq T
\end{aligned}\right.
$$

we find $b_{k}=c_{k}=0, k=1,2,3, \ldots$ and $a_{2 m}=0, a_{2 m-1}=\frac{T p}{\pi^{2}(2 m-1)^{2}}, r_{2 m-1}=a_{2 m-1}, m=$ $1,2,3, \ldots$ Thus all even regions of instability are degenerated and for odd regions we get

$$
\mu^{2} \geq \frac{\pi^{4}(2 m-1)^{4}}{T^{2} p^{2}(1+\alpha T)^{2}}\left[\alpha^{2}+\left(\omega-\frac{(2 m-1) \pi}{T}-\gamma\right)^{2}\right], \quad m=1,2,3, \ldots
$$

from (31). As we see the third, the fifth... regions of instability can occur only with sufficiently large amplitudes of the external force.

Example 6 For the function $\varphi(t)=\cos \theta t$ we obtain $a_{1}=c_{1}=0, b_{1}=r_{1}=\frac{1}{2 \theta}$ and $\theta=\frac{2 \omega}{k}$. Equation $\theta=\frac{2 \omega}{k}(k=1,2,3, \ldots)$ gives the relationship between the frequency of external force and the frequencies of free vibrations of the shell. Near the points the formation of vibrations with unboundedly increasing amplitudes are possible. Since $\theta=2 \omega$ for $k=1$, the parametric resonance takes place when the frequency $\theta$ of the external action is equal to the double eigenvalues of the system. From (31) it follows that

$$
\bar{\mu}^{2} \geq \frac{\alpha^{2}+\gamma^{2}}{r_{1}^{2}}=4 \theta^{2}\left(\alpha^{2}+\gamma^{2}\right) .
$$

In addition to the above mentioned results we may note that if $\theta=2 \omega$ and

(a) if $\bar{\mu}^{2}>4 \theta^{2}\left(\alpha^{2}+\gamma^{2}\right)$ the vibrations are instable; there will be parametric resonance;

(b) if $\bar{\mu}^{2}=4 \theta^{2}\left(\alpha^{2}+\gamma^{2}\right)$ the vibrations are stable and tend to periodic motion;

(c) if $\bar{\mu}^{2}<4 \theta^{2}\left(\alpha^{2}+\gamma^{2}\right)$ the vibrations are asymptotically stable.

Generally, the resonance domain in the space $(\alpha, \omega, \mu)$ consists of the union of half space $\alpha<0$ with the half horn domains on $\alpha \geq 0$ satisfied the conditions $\bar{\mu}^{2} \geq \frac{\alpha^{2}}{r_{k}^{2}}$. Horn domains begin from the points $\omega=k \pi / T$ on $\omega$ axis and incline towards $\alpha 0 \mu$ plane with the increasing viscosity.

\section{Conclusion}

The partial integro-differential equations for dynamic stability of isotropic homogeneous viscoelastic extremely shallow and the circular cylindrical shells with any hereditary properties, including time-dependence of Poisson's ratio, are derived. These equations are reduced to the ordinary integro-differential equation with variable coefficients. Using the Laplace integral transform, the ordinary integro-differential equation is reduced to the new integrodifferential one, the main part of which coincides with damped Hill's equation. The parameter of Hill's equation depends on the Fourier transforms of the hereditary kernel and the frequency of elastic vibrations. The integral term in the obtained integro-differential equation is proportional to the product of two small parameters and tends to zero by time. The monodromy matrix for the integro-differential equation is obtained using the averaging method. Supposing the module of the eigenvalue of the monodromy matrix is greater than the unit, the stability condition is obtained. Analysis of the obtained inequality in the threedimensional space of parameters is carried out. The effect of viscoelasticity on the instability region is investigated and it is found that viscoelasticity increases the stability of the shell. 
Acknowledgements The author acknowledges his indebtedness to Ophelia Hassan for her enthusiastic encouragement during the preparation of the paper.

Open Access This article is distributed under the terms of the Creative Commons Attribution Noncommercial License which permits any noncommercial use, distribution, and reproduction in any medium, provided the original author(s) and source are credited.

\section{References}

Ahmadi, G., Glockner, P.G.: Dynamic stability of Kelvin-Viscoelastic column. J. Eng. Mech. 109(4), 990998 (1983)

Bolotin, V.V.: The Dynamic Stability of Elastic Systems. Holden-Day, San Francisco (1964)

Christensen, R.M.: Theory of Viscoelasticity. An introduction. Academic Press, New York (1971)

Giorgi, G., Lazzai, B.: On the stability for linear viscoelastic solids. Q. Appl. Math. 55, 659-675 (1997)

Iliushin, A.A., Pobedrya, B.E.: The Mathematical Foundation of Thermoviscoelastic Theory. Nauka, Moscow (1970) (in Russian)

Ilyasov, M.H.: Dynamic stability of viscoelastic plates. Int. J. Eng. Sci. 45, 111-122 (2007)

Ilyasov, M.H., Aköz, A.Y.: The vibration and dynamic stability of viscoelastic plates. Int. J. Eng. Sci. 38, 695-714 (2000)

Ilyasov, M.H., Ilyasova, N.M.: Flutter of viscoelastic strips. Mech. Time-Depend. Mater. 10, 201-213 (2006)

Ilyasova, N.M.: Parametric vibrations and resonance. İstanbul Üniv. Fen Fak. Mat. Dergisi 61-62, 41-55 (2002-2003)

Liu, Z., Zheng, S.: On the exponential stability of linear viscoelasticity and thermoviscoelasticity. Q. Appl. Math. 54, 21-31 (1996)

Pata, V.: Exponential stability in linear viscoelasticity. Q. Appl. Math. 64, 499-513 (2006)

Seyranyan, A.P., Mailybaev, A.A.: Multiparameter Stability Theory with Mechanical Applications. World Scientific, Singapore (2003)

Shuping, C., Kangsheng, L., Zhuangyi, L.: Spectrum and stability for elastic systems with global or local Kelvin-Voigt damping. SIAM J. Appl. Math. 59(2), 651-668 (1998)

Tschoegl, N.W., Knauss, W.G., Emri, I.: Poisson's ratio in linear viscoelasticity—a critical review. Mech. Time-Depend. Mater. 6, 3-51 (2002)

Wang, Y.C., Lakes, R.: Stability of negative stiffness viscoelastic systems. Q. Appl. Math. 63, 34-55 (2005) 